\title{
利用序数关系实现相对学习的雾图像能见度检测算法
}

\author{
吴琪 ${ }^{1)}$, 汪小武 ${ }^{1)}$ ，章军 ${ }^{1)}$, 夏懿 ${ }^{1)}$, 陈鹏 ${ }^{2)}$, 阎 (庆 $)^{\text {* }}$ \\ 1) (安徽大学电气工程与自动化学院 合肥 230061) \\ 2) (安徽大学计算机科学与技术学院 合肥 230061)
}

\begin{abstract}
摘 要：针对雾图像的能见度检测在自动驾驶、气象预报等领域有巨大的研究和应用价值，而现有算法忽略了图像 之间的序数关系的问题，提出一种利用序数关系进行相对学习的能见度检测算法. 首先利用数据集中隐藏的序数关 系约束网络学习过程, 并将这种约束简化为图像特征三元组的相对距离关系, 使得图像在特征空间的分布遵循序数 规律; 然后提取训练样本的能见度特征来构造查询库, 通过在特征查询库中寻找近邻图像, 得到测试样本的能见度 检测结果. 在公开的合成雾图像数据集(SF, FROSI)和构建的真实雾数据集(RDF)上进行了验证，实验结果表明，该算 法取得了比现有的多分类、有序多分类等深度学习算法更好的效果, 并且算法在训练过程中更加稳定, 训练数据量要 求更少，具有良好的稳健性和广阔的应用空间.
\end{abstract}

关键词: 能见度和成像; 机器视觉算法; 深度学习; 序数关系; 相对学习

中图法分类号: TP391.41～DOI: 10.3724/SP.J.1089.2020.18250

\section{Ordinal Relation and Relative Learning for Foggy Image Visibility Detection}

\author{
Wu Qi ${ }^{1)}$, Wang Xiaowu ${ }^{1)}$, Zhang Jun ${ }^{1)}, \mathrm{Xia} \mathrm{Yi}^{1)}$, Chen Peng ${ }^{2)}$, and Yan Qing ${ }^{1)^{*}}$ \\ 1) (School of Electrical Engineering and Automation, Anhui University, Hefei 230061) \\ 2) (School of Computer Science and Technology, Anhui University, Hefei 230061)
}

\begin{abstract}
The visibility detection of fog image has great value in the field of automatic driving, weather forecast and so on. However, existing methods ignored the relative information among images. Inspired by this, we propose a deep learning method based on the ordinal relation and triplet relative learning to conduct visibility detection of foggy image. The method uses the ordinal relation hidden in the data to constrain the network learning process and simplifies it to the relative metric of image triplet, which makes the distribution of the image in the feature space follow the law of ordinal rule. The visibility characteristics of the training samples are extracted to construct the query library, and the visibility labels of the test samples are obtained by finding the adjacent images in the feature query library. The method was validated on two public synthesized foggy image dataset(SF/FROSI) and our real dense foggy image dataset(RDF). Experimental results show that the proposed method has better effect than some baseline deep learning methods such as deep multi-classification or ordinal multi-classification. In addition, the method is more stable in the training process and requires less training data, which has excellent robustness and wide application space.
\end{abstract}

收稿日期: 2020-02-26; 修回日期: 2020-10-03. 基金项目：国家自然科学基金青年科学基金(61602002). 吴琪(1994一), 男，硕士 研究生, 主要研究方向为计算机视觉; 汪小武(1964-), 男, 硕士, 副高级工程师, 硕士生导师, 主要研究方向为工业控制、嵌人式系 统开发与研究; 章军(1971一), 男, 博士, 教授, 硕士生导师, 主要研究方向为深度学习在视频分析及化学信息学中的应用; 夏懿 (1976-), 男, 博士, 副教授, 主要研究方向为非平稳信号处理、图像处理与理解、模式识别及深度学习等; 陈鹏 (1978一), 男, 博士, 教授, 博士生导师, 主要研究方向为数据分析及应用、计算机视觉、计算生物学; 阎庆(1978一), 女, 博士, 副教授, 论文通讯作者, 主 要研究方向为模式识别. 
Key words: visibility and imaging; machine vision algorithms; deep learning; ordinal relation; relative learning

雾是一种常见的大气现象. 由于悬浮在大气 中的大量微小水滴、气溶胶等颗粒对光线的散射和 吸收作用, 雾会导致视觉目标的对比度降低、饱和 度下降和色调偏移, 从而影响大气能见度 ${ }^{[1]}$. 能见 度的显著降低, 往往会导致公路、航空和水上运输 的交通事故，造成严重的生命和财产损失. 目前， 大型视频监控系统广泛部署于公共或私人场所, 基于监控摄像头的图像能见度检测算法在气象 学、公共交通等领域具有重要的研究意义和应用 价值.

\section{1 相关工作}

传统的雾图像能见度检测算法 ${ }^{[2-6]}$ 借助人工提 取的图像特征如边缘、颜色、对比度，或大气散射 模型，探索与能见度之间的关系. 如陈到正等 ${ }^{[2]}$ 采 用图像边缘检测算法，提取图像边缘特征点，根据 可见目标的匹配结果估计能见度. $\mathrm{Kim}^{[3]}$ 讨论了大 气消光系数与图像 HSI 色差的非线性关系, 利用色 度分析法对固定场景图像的视觉范围进行估计. Babari 等 ${ }^{[4]}$ 和 Carretas 等 ${ }^{[5]}$ 借助大气散射模型来描述图 像对比度分布与大气能见度之间的映射关系. Graves 等 ${ }^{[6]}$ 根据暗通道先验理论得到大气光值, 进一步推 算能见度值. 这些工作取得了丰硕的研究成果.

但是, 雾图像的视觉效应研究是个复杂过程, 与能见度相关的图像特征和外部条件很多, 包括光 源、天气条件和相机设置等, 很难将所有的相关特 征都提取出来. 其次, 基于 Koschmieder 定律 ${ }^{[7]}$ 提出 的大气散射模型，可以通过计算雾图像的透射率 图或消光系数来获得能见度值. 但 Koschmieder 定 律的成立需要以均质大气为前提条件，而在真实 场景中，雾的分布通常是不均匀的 ${ }^{[8]}$. 因此，基于 传统算法很难取得令人满意的检测结果，也不具 有普遍适用性.

近年来, 利用卷积神经网络(convolutional neural networks, CNN)进行端到端学习的算法, 在图像视 觉领域取得了比传统算法更好的效果 ${ }^{[9]} . \mathrm{Li}^{\text {等 }}{ }^{[10]}$ 对 比了 3 种传统检测算法: 对比度、梯度特征和大气 散射模型，指出手工提取特征及物理模型的方式 很难描述远处物体的可见性, 并首次将 CNN 引人 能见度检测任务, 利用预先训练的 AlexNet ${ }^{[11]}$ 提取 特征进行能见度等级的分类. Giyenko 等 ${ }^{[12]}$ 通过搭
建一个浅层的 CNN(shallow CNN, SCNN) 实现对图 像的能见度检测, 并在闭路摄像机采集的数据集 上取得了比较好的结果. Palvanov 等 ${ }^{[13]}$ 结合传统图 像预处理方法，通过一个深度集成的 $\mathrm{CNN}$ (VisNet), 同时学习原始图像以及经过快速傅 里叶变换或光谱滤波处理的图像, 得到对雾图像 中能见度等级的综合分类.

这些算法都是基于目前最广泛应用的深度多 分类算法 ${ }^{[1]}$, 即 CNN 和逻辑回归函数的结合. 尽 管深度学习的效果明显优于传统算法, 但在雾图 像检测中依然存在一些挑战. (1) 作为一种数据驱 动的算法, 算法性能在很大程度上取决于数据集 的大小和标注质量. 当前, 具有准确能见度标注的 雾图像很难获得. 为此, 收集了一个真实公路场景 下的雾图像数据集(real dense foggy image dataset, $\mathrm{RDF}$ ), 借助专业光学测量仪和气象人员进行注释, 最终得到了较为准确的能见度等级标签. (2) 能见 度检测不是一个简单的分类任务, 不同能见度等 级的雾图像中隐藏着序数关系. 目前直接进行归 纳分类的深度学习算法往往忽略了不同等级图像 之间的序数关系，对数据的利用不够充分. 此外, 受到人类视觉观测法和相对学习 ${ }^{[14]}$ 的启发, 对单 幅图像进行能见度估计是困难的，但是完成不同 能见度图像的对比分析却简单很多. 即通过比较 多幅图像进行能见度检测的方式, 相比现有针对 单幅图像的学习方式, 更加合理高效.

综上所述，传统检测算法提取特征单一、模型 理想化, 而现有深度学习算法存在丢失序数信息 以及只进行单幅图像学习的问题. 针对这些问题, 本文提出了一种基于序数关系和三元组相对学习 的端到端的图像能见度检测算法, 其将雾图像的 分类检测转换为序数问题. 首先, 利用序数关系对 原始数据进行三元重组, 通过相对学习方式提取 图像三元组内的能见度属性, 并将其映射到特征 空间; 然后根据待测图像在特征空间的邻近样本 标签决定检测结果. 相比现有算法, 本文算法充分 利用了雾图像的序数信息, 构造了更加有效的能 见度特征提取过程, 以提高对雾图像能见度的检 测性能. 在合成以及构建的真实雾图像数据集上 的实验表明，本文算法比已有能见度检测算法取 得了更好的检测效果, 同时所需的训练数据量更 少，具有实际应用价值. 


\section{2 算法原理}

\section{1 序数关系}

雾图像的能见度检测可以看做一个序数回归 问题. 如图 1 所示, 每幅图像都有一个标注可见性 等级的标签, 等级之间存在序数关系, 但序数尺度 之间的真实度量距离却是未知的。

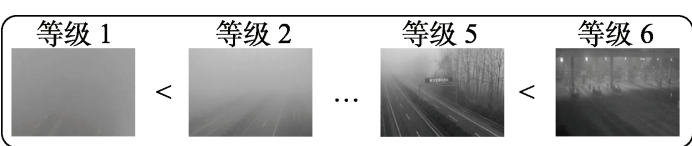

图 1 图像能见度序数关系的示意图

通常解决序数问题有 2 种主要的方式. (1) 通 过顺序编码将有序回归转化为一组嵌套的二分类 问题, 如 Sun 等提出的序数核判别法 ${ }^{[15]}$ 和序数向量 量化法 ${ }^{[16]}$. (2) 将序数回归转化为正则回归, 如 Kramer 等 ${ }^{[17]}$ 通过回归树学习器将序数尺度映射到 连续数值, 其关键是找到一个合适的映射函数来 实现离散数据的连续化.

近年来，深度学习算法已被应用于解决序数问 题. Niu 等 ${ }^{[18]}$ 将人脸年龄估计任务看做序数问题，利 用多输出的 $\mathrm{CNN}$ (multiple output $\mathrm{CNN}, \mathrm{MOCNN}$ ) 解决序数多二分类问题. $\mathrm{Fu}$ 等 ${ }^{[19]}$ 提出了一种间距递 增离散化(spacing increasing discretization, SID)策 略, 将深度任务转换为序数回归问题. Coh 等 ${ }^{[20]}$ 提出 一种基于成对的正则回归算法(CNN with pairwise regularization for ordinal regression, CNNPOR), 假 定相邻秩回归值之间的最小间隔为 1 , 并将其作为 一种度量约束与交叉熵损失相结合，共同学习一个 满意的序数回归函数. 这些研究成果表明, 利用序 数信息有助于深度学习任务的实现.

\section{2 相对学习}

现有的深度学习算法大多是在单个属性标签 的约束下从图像中提取需要的特征. Parikh 等 ${ }^{[14]}$ 提 出一种对数据之间某种特定属性的强度进行比较 的学习方式, 使得数据之间可以根据它们的属性 相互关联起来，即相对学习. Souri 等 ${ }^{[21]}$ 将深度学 习模型与相对属性结合, 用 CNN 学习图像对的某 些相似或不同强度的特定属性, 并取得了比支持 向量机 ${ }^{[14]}$ 和相对森林 ${ }^{[22]}$ 更好的结果.

能见度也是一种相对属性，它可以被投影到 某个特征空间. 如图 2 所示, 如果原图像三元组 $\left(x_{i}, x_{j}, x_{k}\right)$ 的能见度 $\left(v_{i}, v_{j}, v_{k}\right)$ 满足序数关系 $v_{i}<$ $v_{j}<v_{k}$, 则当将图像的能见度属性投影到某个特 征空间后，相应特征的分布也应当满足该规律. 即 原始图像的能见度差异 $D_{V}\left(x_{i}, x_{j}\right)$ 较大时, 在投影 空间中特征的相对距离 $D_{F}\left(x_{i}, x_{j}\right)$ 也较大. 该关系 可表示为

$$
\left\{\begin{array}{ll}
D_{F}\left(x_{i}, x_{j}\right)<D_{F}\left(x_{i}, x_{k}\right), & \text { if } D_{V}\left(x_{i}, x_{j}\right)<D_{V}\left(x_{i}, x_{k}\right) . \\
D_{F}\left(x_{i}, x_{j}\right) \geqslant D_{F}\left(x_{i}, x_{k}\right), & \text { otherwise }
\end{array} .\right.
$$

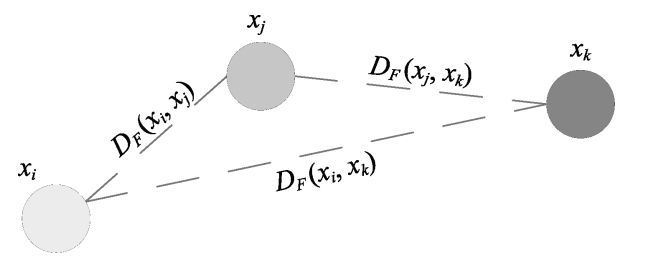

图 2 特征空间中三元组相对关系的示意图

\section{3 序数关系和相对学习的能见度检测}

如第 2.1 节所述，尽管能见度等级之间的差异 已知，但真实能见度 $v_{i}$ 之间的度量距离是未知的. 因此在 FaceNet ${ }^{[23]}$ 等算法中人为规定特征之间的距 离为常数, 即令 $D_{F}\left(x_{i}, x_{j}\right)=\alpha$, 这显然是不合理 的. 而利用属性之间的序数关系进行相对比较的 方式，样本的特征分布更加自然，可以获得对整个 数据集的能见度属性的序数度量. 本文算法的实 现过程如图 3 所示.

\section{1 改进的 CNN}

本文的雾图像深度 CNN (foggy CNN, FCNN)是 对经典的图像识别 CNN (visual geometry group 16, VGG16) ${ }^{[2]]}$ 进行改进, 其网络结构如图 4 所示. 相比 于 VGG16 直接将图像缩小为 $224 \times 224 \times 3, \mathrm{FCNN}$ 在输人环节增加填充层和卷积层, 将原始图像转 换为 $224 \times 224 \times 3$ 的特征图, 以充分保留雾图像中 有助于能见度检测的浅层纹理特征. 第 2 第 14 卷 积层加载 ImageNet 上预训练的 VGG16 参数, 作为 初始化值进行下一步微调. 在每个卷积层和激活 函数之间加人批标准化手段 ${ }^{[25]}$ 充当正则化器, 并 使模型对初始化值不敏感. 最后将卷积网络输出 的特征图展平为向量, 经过 4 层全连接层进一步降 维, 并在 1 3 全连接层后加人抑制层(Dropout) $)^{[26]}$ 预防过拟合, 最终得到维度为 $d$ 的能见度属性. 和 VGG16 相比, FCNN 的参数量基本不变, 但选择性 地保留了更多的浅层纹理特征. 


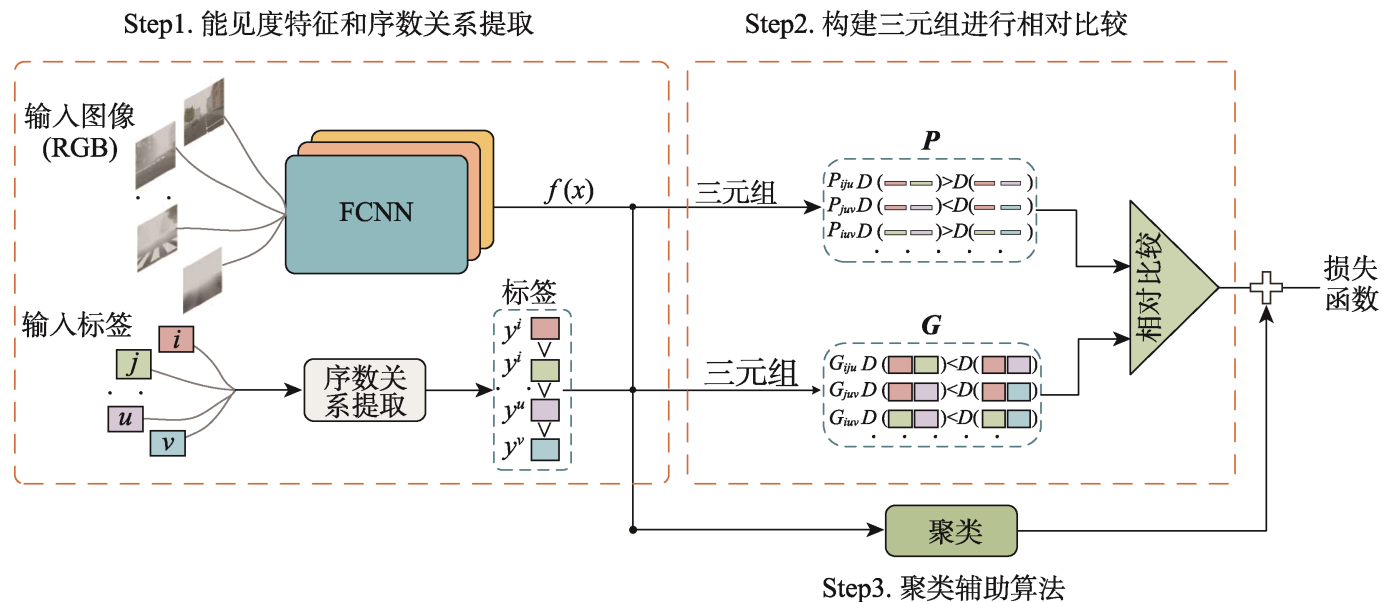

图 3 算法实现流程图

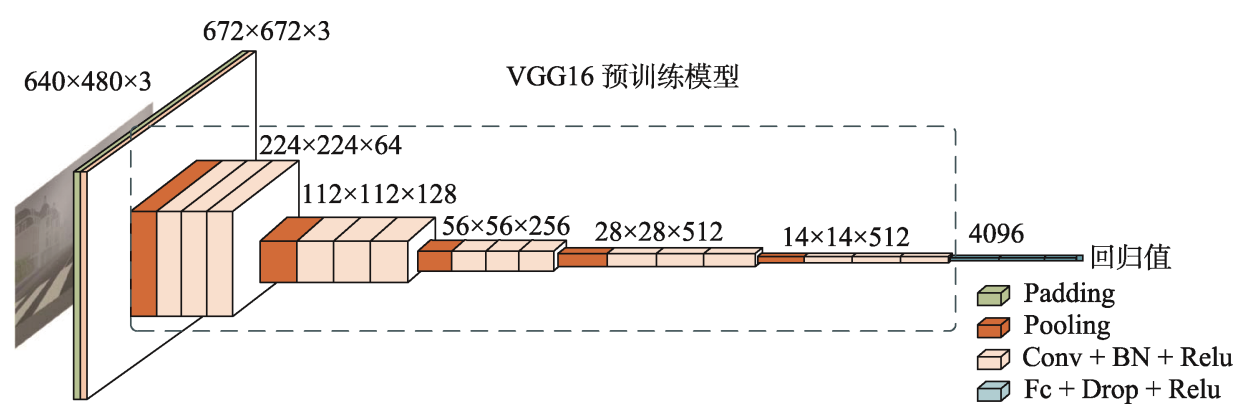

图 $4 \mathrm{FCNN}$ 结构图

\section{2 序数三元组相对比较}

\subsection{1 序数三元组重构}

假设雾图像数据集 $(X, Y)$ 中包含 $M$ 个能见度 级别, 即 $Y=\{1,2, \cdots, M\}$. 用

$$
T^{m}=\left\{\left(x_{i}^{m}, y_{i}^{m}\right) \mid x_{i}^{m} \in X, y_{i}^{m} \in Y\right\}
$$

表示第 $m$ 类的训练样本子集. 为了简化表示, 进一 步用 $\left(x^{m}, y^{m}\right)$ 取代 $\left(x_{i}^{m}, y_{i}^{m}\right)$ 表示 $T^{m}$ 中的任一样本.

由于原始图像的能见度值 $v_{i}$ 未知, 只定义图 像之间能见度等级的差异 $D_{Y}$ 和投影空间上特征的 距离 $D_{F}$, 分别为

$$
\begin{aligned}
& D_{Y}\left(x^{m}, x^{u}\right)=\left|y^{m}-y^{u}\right|, \\
& D_{F}\left(x^{m}, x^{u}\right)=\left\|f\left(x^{m}\right), f\left(x^{u}\right)\right\|_{2}^{2} .
\end{aligned}
$$

其中, $\left(x^{m}, y^{m}\right) \in T^{m},\left(x^{u}, y^{u}\right) \in T^{u} ; f(\cdot)$ 为原始图 像到特征空间的投影函数. 按照由低到高的标注方 式，各等级雾图像之间满足 $y^{1}<\cdots<y^{m}<\cdots<y^{M}$, 并可进一步表示为能见度差异 $D_{Y}$ 的序数关系, 即

$$
D_{Y}\left(x^{1}, x^{2}\right)<\cdots<D_{Y}\left(x^{1}, x^{m}\right)<\cdots<D_{Y}\left(x^{1}, x^{M}\right)
$$

式(1)将序数问题转换为 $M$ 元组的相对关系, 并可进一步等效为一系列序数三元组的相对位置 关系(如图 2 所示), 即

$$
\left\{\begin{array}{c}
D_{F}\left(x^{1}, x^{2}\right)<D_{F}\left(x^{1}, x^{3}\right) \\
D_{F}\left(x^{2}, x^{3}\right)<D_{F}\left(x^{2}, x^{4}\right) . \\
\ldots
\end{array} .\right.
$$

在一个包含 $R$ 幅图像的训练批次中, 理论上 能够得到 $R^{3}$ 个三元组. 但只有不同等级图像之间 的序数关系可知, 并被定义为序数三元组集合 $U=\left\{\left(x_{i}, x_{j}, x_{k}\right) \mid y_{i} \neq y_{j} \neq y_{k}\right\}$. 原始空间下, 集合内 三元组的相对关系 $g_{(i j k)}$ 可以表示为

$$
\begin{aligned}
& g_{(i j k)}=\left\{\begin{array}{ll}
1, & \text { if } D_{Y}\left(x_{i}, x_{j}\right)<D_{Y}\left(x_{i}, x_{k}\right) \\
0, & \text { otherwise }
\end{array},\right. \\
& \text { s.t. }\left(x_{i}, x_{j}, x_{k}\right) \in U \text {. }
\end{aligned}
$$

\subsection{2 相对比较损失}

要使能见度属性在特征空间的分布满足原始 图像序数规律, 一个简单的方式是使三元组在特 征空间的相对关系 $p_{(i j k)}$ 与原始图像的 $g_{(i j k)}$ 保持一 致, 即

$$
\begin{gathered}
\max \sum_{\left(x_{i}, x_{j}, x_{k}\right) \in U} I\left(g_{(i j k)}, p_{(i j k)}\right), \\
\text { s.t. } I=\left\{\begin{array}{l}
1, \text { if } g_{(i j k)}=p_{(i j k)} \\
0, \text { otherwise }
\end{array}\right.
\end{gathered}
$$

但是这样简单定义 $p_{(i j k)}=0 / 1$ 的方式, 并不能 
很好地建立图像之间的能见度关联. 为此, 定义三 元组 $(i, j, k)$ 在特征空间的相对关系 $p_{(i j k)}$ 为

$$
p_{(i j k)}=\frac{1}{1+\exp \left\{o_{(i j k)}\right\}}
$$

其中, $o_{(i j k)}=D_{F}\left(x_{i}, x_{j}\right)-D_{F}\left(x_{i}, x_{k}\right)$ 表示三元组中 属性之间的距离差异; $p_{(i j k)}$ 是借助 Sigmoid 函数 实现对 $o_{(i j k)}$ 的归一化. 如果 $D_{F}\left(x_{i}, x_{j}\right)<D_{F}\left(x_{i}, x_{k}\right)$, $p_{(i j k)} \rightarrow 1$; 否则, $p_{(i j k)} \rightarrow 0$, 以此保持与式(2)相同 的序数关系. 为了衡量 $p_{(i j k)}$ 与 $g_{(i j k)}$ 的一致性, 用 似然函数定义相对比较的损失为

$$
\begin{aligned}
L_{s}= & -\frac{1}{n} \sum_{\left(x_{i}, x_{j}, x_{k}\right) \in U}\left[g_{(i j k)} \log p_{(i j k)}+\right. \\
& \left.\left(1-g_{(i j k)}\right) \log \left(1-p_{(i j k)}\right)\right] .
\end{aligned}
$$

其中, $n$ 表示 $U$ 中序数三元组的数量.

借助似然函数损失，使网络提取到满足 $\boldsymbol{P}=\boldsymbol{G}$ 的特征. 该特征保持了与雾图像原始空间中 雾能见度等级相一致的序数关系属性. 同时, 由于 式(2)中使用了 Sigmoid 函数, $p_{(i j k)}$ 对 $o_{(i j k)}$ 超过 $[-5$, 5]的部分并不敏感，使得等级差异大的图像在特 征空间的距离更远，将三元组内的相对关系进一 步延伸到整个数据集, 实现特征的序数分布. 在后 续的实验环节中，将展示经三元组相对学习的整 个数据集的能见度属性在二维空间的分布，以表 明本文算法实现了全局的能见度关联。

\section{3 聚类损失}

通过网络训练逐步减小比较损失 $L_{s}$, 网络使 特征的空间分布逐渐满足序数规律. 由式(2)可知, 当 $p_{(i j k)} \rightarrow 1$, 特征间的度量距离 $o_{(i j k)}$ 会随比较损失 $L_{s}$ 的减小而逐渐增大, 特征趋于分散; 但过于分 散的特征分布并不利于最终分类. 为此, 加人一个 轻量级的聚类损失 ${ }^{[27]}$

$$
\begin{gathered}
L_{c}=\frac{1}{2 N} \sum_{m=1}^{M} \sum_{i=1}^{N^{m}}\left\|f\left(x_{i}^{m}\right)-c^{m}\right\|_{2}^{2}, \\
\text { s.t. } c^{m}=\frac{1}{N^{m}} \sum_{i=1}^{N^{m}} f\left(x_{i}^{m}\right) .
\end{gathered}
$$

其中, $N$ 是每个训练批次的图像总数量; $N^{m}$ 是第 $m$ 等级的图像数量; $c^{m}$ 为 $m$ 等级训练样本所对应 的类别中心，在每次迭代中进行更新. 该损失项在 加快网络收玫的同时，限制能见度属性的分布范 围，便于在测试环节查询待测样本的相似图像.

在相对损失和聚类损失的基础上，定义本文 算法总的网络损失函数为 $L=L_{s}+\lambda L_{c}$. 其中, $\lambda$
是 $[0,1]$ 的权重参数, 用于平衡 2 个损失的比例, 在 本文中设置为 0.001

\section{4 基于最近邻的投票机制}

算法在训练环节提取图像的能见度属性, 并 使之在特征空间的分布上遵循已知序数规律, 最 终检测结果通过基于特征空间的近邻算法来确定.

首先，基于训练集 $T$ 中样本 $x_{i}$ 的能见度属性 $f\left(x_{i}\right)$ 和标签 $y_{i}$, 构建查询库 $Q=\left\{\left(y_{i},\left(f\left(x_{i}\right)\right) \mid\right.\right.$ $\left.\left(x_{i}, y_{i}\right) \in T\right\}$. 在检测环节, 从 $Q$ 中寻找与待测样本 $x_{q}$ 的能见度属性 $f\left(x_{q}\right)$ 近邻的 $s$ 个样本集合 $N_{s}$, 最终根据近邻样本集合 $N_{s}$ 投票确定待测样本的标 签 $y_{q}^{\prime}$, 即

$$
\begin{gathered}
y_{q}^{\prime}=\arg \max \sum_{\left(x_{i}, y_{i}\right) \in N_{s}}^{m=1} I\left(y^{m}, y_{i}\right), \\
\text { s.t. } I(x, y)=\left\{\begin{array}{l}
1, \text { if } x=y \\
0, \text { otherwise }
\end{array} .\right.
\end{gathered}
$$

\section{4 实验与分析}

为了验证本文算法的有效性，在公开的雾天 公路标志数据集(foggy road sign images, FROSI) ${ }^{[28]}$ 、 合成雾数据集 (synthesized foggy, SF ${ }^{[29]}$, 以及构造 的真实数据集 RDF 上进行实验; 并与当前广泛应用

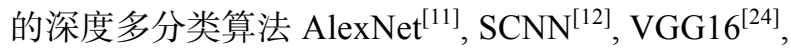
$\mathrm{VisNet}^{[13]}$, 以及序数回归算法 $\mathrm{MOCNN}^{[18]}$ 和 PORCNN ${ }^{[20]}$ 的结果进行了比较.

\section{1 数据集与评价指标}

\subsection{1 数据集}

(1) FROSI 数据集. 一个具有简单道路场景的 人工合成雾图像数据集，能见度从 50 400 m 被分 为 7 个等级, 共 3528 幅, 分辨率 $1400 \times 600$. 每类 选取 70\%作为训练, 30\%作为测试.

(2) SF 数据集. 一个包含 84 个不同道路场景 的合成雾䨪图像数据集, 分为 9 个雾䨪等级, 其中 等级 1 的能见度水平最低, 等级 9 的能见度最高, 共 3024 幅, 分辨率 $640 \times 480$. 每类选取 $70 \%$ 作为 训练, 30\%作为测试.

(3) RDF 数据集. 图像数据由分布于安徽省内 84 个高速公路监控摄像头记录的真实雾图像场景 组成, 分辨率为 $640 \times 480$. 初始的能见度标注来自 摄像头 $1 \mathrm{~km}$ 范围内的气象站点的能见度仪观测结 果. 由于雾这种天气现象具有季节性, 因此在秋冬 季进行数据的采集工作. 2017-10-2018-04, 共采 集到 3 万多幅原始图像和能见度仪标注. 由于雾是 
不断变化和不均匀的，即使专业的能见度仪，仍然 存在许多明显的错误标注. 在专业气象人员的帮 助下, 以能见度仪的初始观测值为参考, 为每幅图 像标注能见度等级, 最终筛选得到 5165 幅满足要 求的图像. 根据大雾预报等级和交通部门的实际需 求, 这些图像被分为 6 个等级, 能见度由低到高分 别为 $0 \sim 0.05 \mathrm{~km}, 0.05 \sim 0.1 \mathrm{~km}, 0.1 \sim 0.2 \mathrm{~km}, 0.2 \sim 0.5 \mathrm{~km}$,
$0.5 \sim 1.0 \mathrm{~km}$ 和 $>1.0 \mathrm{~km}$. 由于大雾是一种罕见的天 气现象, 不同等级的雾图像数量并不均衡. 因此, 在每个类别中随机选取 150 幅(第 1 类约 $50 \%$ )组成 一个平衡的测试集，剩余部分作为训练.

这 3 个数据集的部分图像如图 5 所示, 各能见 度等级图像的数量如表 1 所示. 其中, 标签值越大, 表示图像的能见度等级越高, 雾的浓度越浅.

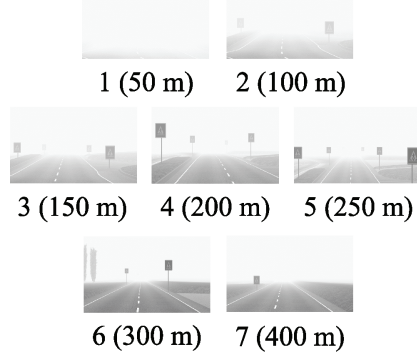

a. FROSI

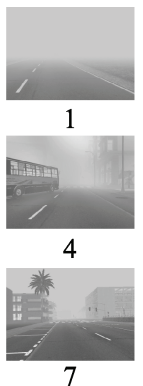

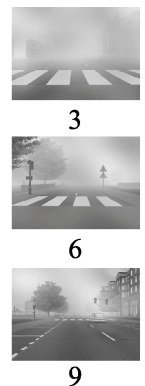

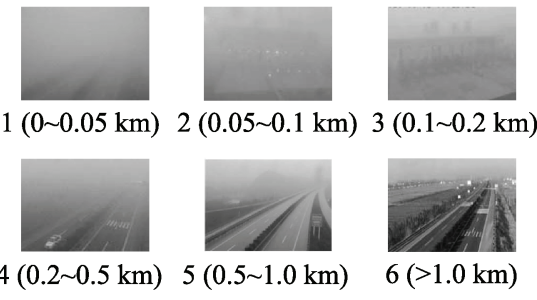

c. RDF

图 5 雾图像数据集中的部分实例

表 13 个数据集中各能见度等级图像的数量 幅

\begin{tabular}{|c|c|c|c|c|c|c|c|c|c|c|}
\hline \multirow{2}{*}{ 数据集 } & \multicolumn{9}{|c|}{ 能见度等级 } & \multirow{2}{*}{ it } \\
\hline & 1 & 2 & 3 & 4 & 5 & 6 & 7 & 8 & 9 & \\
\hline FROSI & 504 & 504 & 504 & 504 & 504 & 504 & 504 & & & 3528 \\
\hline $\mathrm{SF}$ & 336 & 336 & 336 & 336 & 336 & 336 & 336 & 336 & 336 & 3024 \\
\hline RDF & 308 & 625 & 774 & 893 & 951 & 1614 & & & & 5165 \\
\hline
\end{tabular}

\subsection{2 评估指标}

本文采用分类准确率(classification accuracy, ACC)和平均绝对误差(mean absolute error, MAE) 作为评价指标, 对算法性能和稳健性进行评估. ACC 表示能见度预测正确的样本数所占百分比, MAE 代表预测错误的样本与真实值之间的平均误 差距离.

\section{2 实验细节}

本文算法和对比算法均使用 TensorFlow 实现, 并在 1 块 NVIDIA 1080Ti 显卡上运行. 模型训练均 采用 Adam 优化器, 单个训练批次的样本数量根据 GPU 的显存上限设置: 在 VGG, FCNN, MOCNN, PORCNN 和本文算法中均设为 48 , 在 AlexNet, $\mathrm{SCNN}, \mathrm{VisNet}$ 中设为 24. 在 FROSI 和 SF 数据集 上的初始学习率设为 0.00001 , 衰减系数设为 $0.95 / 200$ 次; 在 RDF 数据集上的初始学习率设为 0.0001 , 衰减系数设为 $0.95 / 400$ 次. 所有模型均训 练 200 个 epoch.

为了使实验结果更加客观，本文对每种算法 均独立实验 5 次, 每次随机划分训练和测试样本, 计算 5 次实验的评价指标的均值.

\section{3 实验结果与分析}

\subsection{1 超参数分析}

由于本文提出的模型中有 3 个参数: 目标函数 权重 $\lambda$ 、特征维度 $d$ 和近邻算法的参数 $s$. 为了找 到比较可靠的参数组合, 先单独分析每个参数对 模型的影响程度, 找到各参数的合理区间; 再在此 基础之上，进一步得到最优组合.

首先固定参数 $d=8$ 和 $s=50$, 研究 $\lambda$ 对模型 结果的影响. 如表 2 所示, 不同的 $\lambda$ 会对分类结果 产生正面或负面影响. 当加人轻微的聚类损失 $(\lambda=0.0001,0.001)$ 时, 能够显著地提高模型的分 类效果, 但当加人的聚类损失比重较大 $(\lambda=0.01)$ 时, 会大幅度降低模型的精度. 因为轻微的聚类损 失可以约束特征之间的分布，使之更加合理，而过 大的聚类损失会使特征在空间内的整体分布趋于 汇聚，不利于检测阶段 $K \mathrm{NN}$ 算法对不同类别样本 的区分. 因此, 在后面的实验中, 统一设置 $\lambda=0.001$.

表 3 展示了不同特征维度 $d$ 下的实验结果. 其

表 2 不同 $\lambda$ 下的实验数据对比

\begin{tabular}{cccccc}
\hline \multirow{2}{*}{$\lambda$} & \multicolumn{3}{c}{$\mathrm{SF}$} & \multicolumn{2}{c}{$\mathrm{RDF}$} \\
\cline { 2 - 3 } \cline { 5 - 6 } & $\mathrm{ACC} / \%$ & $\mathrm{MAE}$ & & $\mathrm{ACC} / \%$ & $\mathrm{MAE}$ \\
\hline $10^{0}$ & 96.44 & 0.035 & & 83.50 & 0.162 \\
$10^{-4}$ & 96.78 & 0.031 & & 85.55 & 0.156 \\
$10^{-3}$ & $\mathbf{9 7 . 1 4}$ & $\mathbf{0 . 0 2 6}$ & $\mathbf{8 7 . 3 7}$ & $\mathbf{0 . 1 2 9}$ \\
$10^{-2}$ & 76.67 & 0.280 & 82.44 & 0.183 \\
\hline
\end{tabular}

注. 加粗数值表示最好结果. 
中, 低维度特征 $\left(d=2^{1} / 2^{2}\right)$ 的结果明显优于高维度 特征 $\left(d=2^{5} / 2^{6}\right)$. 说明在高维特征中存在更多没能 正确分布的样本，影响了 $K \mathrm{NN}$ 近邻查找的效果. 即利用标签的序数关系, 对低维特征的序数分布 约束更加有效.

表 3 不同 $d$ 下的实验数据对比

\begin{tabular}{cccccc}
\hline \multirow{2}{*}{$d$} & \multicolumn{3}{c}{$\mathrm{SF}$} & & \multicolumn{2}{c}{$\mathrm{RDF}$} \\
\cline { 2 - 3 } \cline { 5 - 6 } & ACC $\%$ & MAE & & ACC $\%$ & MAE \\
\hline $2^{1}$ & $\mathbf{9 7 . 3 3}$ & $\mathbf{0 . 0 2 5}$ & & 87.44 & 0.132 \\
$2^{2}$ & 97.15 & 0.027 & & $\mathbf{8 7 . 6 8}$ & $\mathbf{0 . 1 2 5}$ \\
$2^{3}$ & 97.14 & 0.026 & & 87.37 & 0.129 \\
$2^{4}$ & 97.13 & 0.031 & & 87.35 & 0.130 \\
$2^{5}$ & 96.92 & 0.032 & & 87.28 & 0.132 \\
$2^{6}$ & 96.61 & 0.037 & & 86.13 & 0.143 \\
\hline
\end{tabular}

注. 加粗数值表示最好结果.

图 6 进一步展示不同 $s$ 下的实验结果, 可以看 到, $s$ 对模型的影响有限, 也说明模型的最终分类 结果是比较稳定的. 最终, 根据超参数实验选取最 佳组合：在 SF 数据集中 $\lambda=0.001, d=2, s=10$; 在 RDF 数据集中 $\lambda=0.001, d=4, s=10$, 并与其他算 法的结果进行比较.
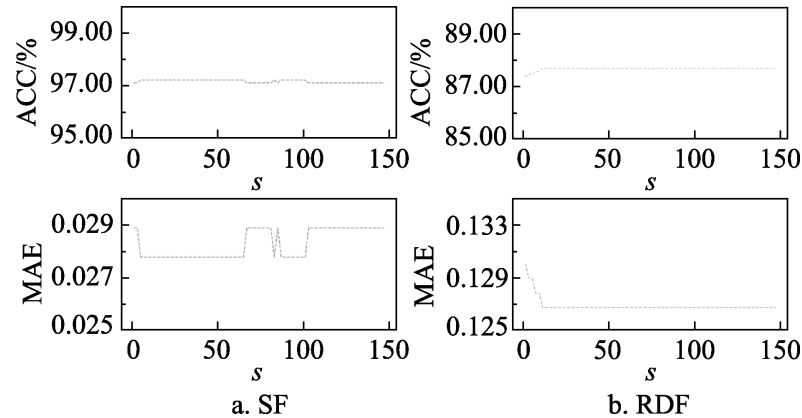

图 62 个数据集中不同 $s$ 的准确率

\subsection{2 对比实验分析}

本文算法的创新主要包括改进的 FCNN 和序 数相对学习 2 个方面. 为探究两者对能见度检测任 务的影响, 将对比实验分为 2 个层次, 实验结果如 表 4 所示. 由于 FROSI 数据集结构较简单，在基于 改进的 FCNN 下，多个算法均实现了 $100 \%$ 的分类 准确率.

第 1 个层次，基于当前深度多分类算法，对比 不同卷积网络的检测效果. 即利用 CNN 和逻辑回 归函数进行损失计算和分类检测的算法，如 AlexNet, SCNN, VGG16, VisNet 和 FCNN 等. 其中, VGG16 和 FCNN 加载了 ImageNet 预训练后的模型 参数作为初始化. 很显然, 本文设计的 FCNN 取得
了最好的检测效果，证明了本文 FCNN 更适合雾 图像的能见度检测任务; 同时说明, 保留图像中更 多的浅层纹理特征，是有利于能见度检测任务的.

表 4 不同算法在 3 个数据集上的实验结果

\begin{tabular}{|c|c|c|c|c|c|c|}
\hline \multirow{2}{*}{ 算法 } & \multicolumn{2}{|c|}{ FROSI } & \multicolumn{2}{|c|}{ SF } & \multicolumn{2}{|c|}{ RDF } \\
\hline & $\mathrm{ACC} / \%$ & MAE & $\mathrm{ACC} / \%$ & MAE & $\mathrm{ACC} / \%$ & MAE \\
\hline AlexNet ${ }^{[11]}$ & 98.27 & 0.018 & 70.41 & 0.323 & 69.41 & 0.344 \\
\hline $\mathrm{SCNN}^{[12]}$ & 98.76 & 0.015 & 62.86 & 0.412 & 70.55 & 0.363 \\
\hline VGG16 $6^{[24]}$ & 98.40 & 0.017 & 88.08 & 0.114 & 83.64 & 0.181 \\
\hline VisNet $^{[13]}$ & 98.60 & 0.014 & 86.97 & 0.144 & 84.16 & 0.179 \\
\hline FCNN & 100.00 & 0.000 & 94.09 & 0.059 & 85.89 & 0.162 \\
\hline $\operatorname{MOCNN}^{[18]}$ & 100.00 & 0.000 & 94.65 & 0.052 & 86.44 & 0.150 \\
\hline $\mathrm{PORCNN}^{[20]}$ & 100.00 & 0.000 & 94.68 & 0.049 & 86.05 & 0.102 \\
\hline 本文 & 100.00 & 0.000 & 97.55 & 0.024 & 87.68 & 0.125 \\
\hline
\end{tabular}

注. 加粗数值表示最好结果.

第 2 个层次，对基于深度多分类算法的 FCNN、基于序数回归算法的 MOCNN 和 PORCNN, 以及基于序数相对学习的本文算法进行比较. 这 4 种算法的卷积网络均采用 FCNN, 超参数设置也完 全一致. 从表 4 对比结果可以看出, 相比现有深度 分类算法, 添加了序数信息的 $\mathrm{MOCNN}$, PORCNN 和本文算法各项指标均有明显的提高.

同时，本文算法在各项指标都取得了比另 2 种 序数回归算法更好的性能. 这是因为 MOCNN 借 助序数信息来学习一系列嵌套的二分类器, 实际 上还是一种添加了序数信息的深度多分类算法. 而 PORCNN 仅学习相邻类之间的成对约束, 对数 据集中序数信息的利用不够充分，并且这种将相 邻类秩之间的距离手工设置为 1 的方式, 可能与数 据集的真实度量相偏离. 相比而言, 本文将图像编 码为一系列序数三元组, 并对图像之间的能见度 大小关系进行比较的学习方式, 对序数信息的利 用更加充分. 通过学习图像之间的能见度差异, 网 络能够更准确地获得图像中的能见度特征, 并对 整个数据集的能见度大小进行全局排序.

在 SF 数据集和 RDF 数据集上, 网络提取的能 见度属性在二维空间上的分布如图 7 所示. 可以看 到, 雾图像的能见度属性按照等级依次排列.

图 8 展示了 $\mathrm{SF}$ 和 $\mathrm{RDF}$ 测试集上的一些测试实 例(带边框的图像表示预测错误的实例), 并在每个 实例的左上角给出标签值和网络预测结果. 这些 实例 (特别是低能见度的图像)通过肉眼很难判断 出真实能见度, 而机器视觉算法能够发掘雾图像 之间像素的细微变化，并找到能见度规律. 


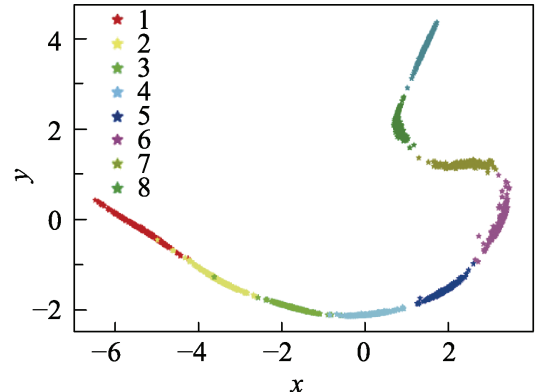

a. SF数据集

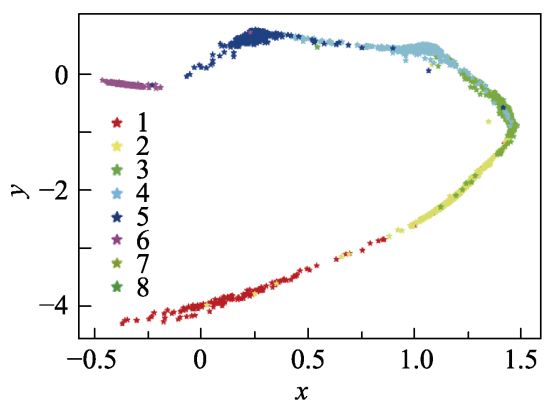

b. RDF数据集

图 72 个数据集中能见度属性的空间分布图
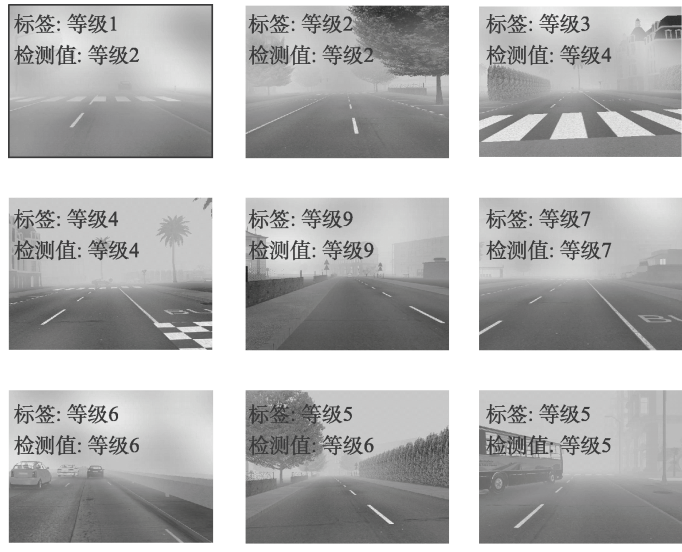

尽管有少量图像预测错误，其预测值和真实 值的误差范围也都在 1 个等级以内. 对于气象预报 和交通管控等实际应用场景来说, 这样的误差范 围是可以接受的. 为此, 统计了预测结果的绝对误 差在一个等级内的准确率为 $99.78 \%$, 已经非常接 近 $100.00 \%$, 能够满足实际气象应用的需求，具有 实际使用价值.

由于获取真实场景中的能见度标注图像比较 困难，在实际中可能面临小数据集的应用要求. 为此, 在平衡的数据集 $\mathrm{SF}$ 上选取不同的训练集比 例, 以验证算法对小数据集的适用性. 从图 9 可以 看出, 在训练数据比例大幅度降低的情况下, 本文 算法的准确率下降趋势最小, 并仍保持 $90.00 \%$ 以 上的分类精度. 说明本文这种图像之间进行相对 比较的学习方式, 能够有效地提高网络的特征提 取效率, 且训练过程所需数据量相对较少, 进一步 说明本文算法的应用价值. 对于一些数据收集同 样困难的深度学习任务来说, 同样具有意义.
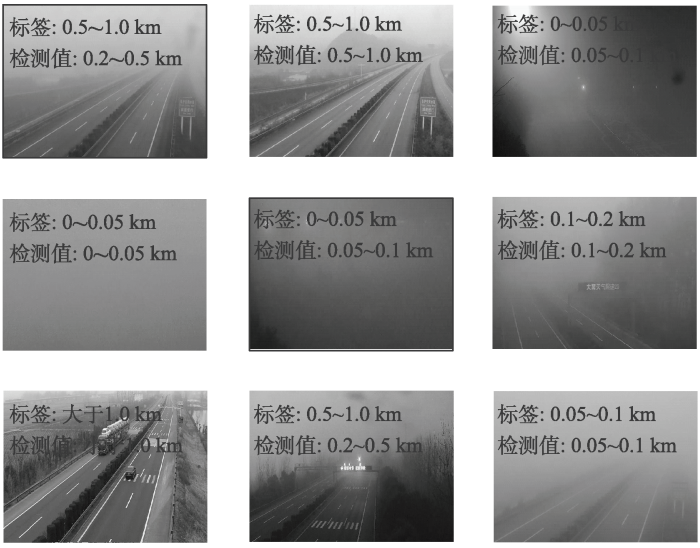

图 8 2 个数据集中部分测试实例展示

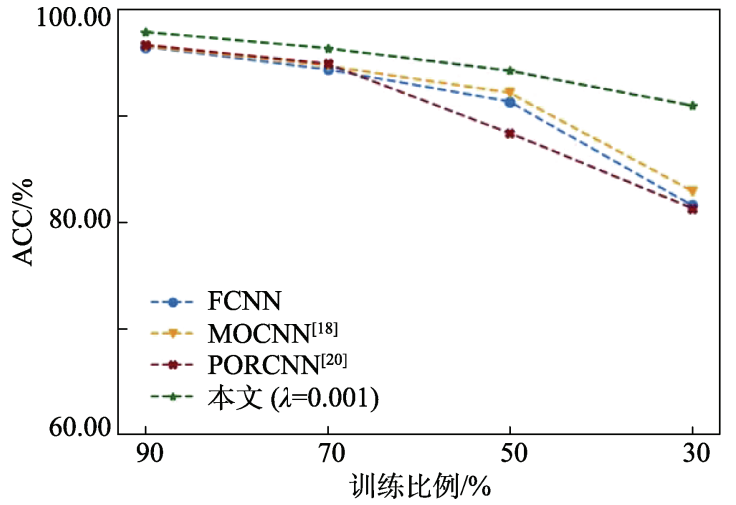

图 $9 \mathrm{SF}$ 数据集中不同训练比例下的准确率曲线

\subsection{3 各能见度等级的分析}

$\mathrm{SF} / \mathrm{RDF}$ 数据集在各能见度类别上的检测结果 分别如表 5 和表 6 所示. 可以看出, 所有基于深度
学习的算法在浓雾下的检测精度均明显低于浅雾, 即呈现出能见度越高, 检测结果越好的趋势. 这是 因为与轻雾图像相比, 浓雾图像中大气散射现象 更加严重, 雾图像中保留的结构特征较少, 能见度 估计难度大。

相对于其他深度学习算法, 本文算法能较好 地处理低能见度图像. 由表 5 和表 6 的结果可以发 现，本文算法在低能见度(标签为 1 4)时保持了较 高的分类精度，与同类算法相比优势明显. 主要原 因是，在浓雾情况下，因为缺乏可以利用的图像特 征，能见度信息检测的效果会更加依赖于对序数 关系的表征. 而本文算法正是通过对序数关系的 有效分析, 更准确地提取图像中的能见度属性, 提 高了雾图像能见度的识别效果. 
表 5 SF 数据集上各算法在不同能见度等级下 准确率比较

\begin{tabular}{lccccccccc}
\hline \multirow{2}{*}{ 算法 } & \multicolumn{8}{c}{ 能见度等级 } \\
\cline { 2 - 9 } & 1 & 2 & 3 & 4 & 5 & 6 & 7 & \multicolumn{1}{c}{8} & \multicolumn{1}{c}{9} \\
\hline AlexNet $^{[11]}$ & 64.0 & 44.0 & 57.0 & 58.5 & 70.0 & 76.0 & 79.0 & 86.0 & 93.5 \\
SCNN $^{[12]}$ & 53.5 & 28.5 & 44.5 & 57.5 & 43.5 & 54.0 & 65.5 & 82.5 & 93.5 \\
VGG16 $^{[24]}$ & 67.0 & 81.5 & 80.5 & 85.5 & 87.5 & 89.5 & 94.0 & 96.0 & 98.5 \\
$\operatorname{VisNet}^{[13]}$ & 72.0 & 75.0 & 83.0 & 86.5 & 87.5 & 89.5 & 94.0 & 98.5 & $\mathbf{1 0 0 . 0}$ \\
FCNN & 79.3 & 91.7 & 94.0 & 95.0 & 95.7 & 96.0 & 99.0 & $\mathbf{1 0 0 . 0}$ & 99.7 \\
MOCNN $^{[18]}$ & $\mathbf{9 0 . 0}$ & 81.7 & 93.3 & 92.7 & 96.3 & 99.0 & 99.3 & 99.7 & 99.7 \\
PORCNN $^{[20]}$ & 85.2 & 85.8 & 93.3 & 95.7 & 95.2 & 98.0 & 99.0 & 99.5 & 99.8 \\
本文 & $\mathbf{9 0 . 0}$ & $\mathbf{9 5 . 3}$ & $\mathbf{9 6 . 7}$ & $\mathbf{9 7 . 3}$ & $\mathbf{9 8 . 0}$ & $\mathbf{9 9 . 3}$ & $\mathbf{9 9 . 7}$ & $\mathbf{1 0 0 . 0}$ & 99.7 \\
\hline
\end{tabular}

注. 加粗数值表示最好结果.

表 6 RDF 数据集上各算法在不同能见度等级下 准确率比较

\begin{tabular}{lcccccc}
\hline \multirow{2}{*}{ 算法 } & \multicolumn{6}{c}{ 能见度等级 } \\
\cline { 2 - 7 } & \multicolumn{1}{c}{1} & \multicolumn{1}{c}{2} & \multicolumn{1}{c}{3} & \multicolumn{1}{c}{4} & \multicolumn{1}{c}{5} & 6 \\
\hline AlexNet $^{[11]}$ & 0.0 & 80.3 & 75.6 & 79.3 & 83.8 & 97.6 \\
SCNN $^{[12]}$ & 1.3 & 75.3 & $\mathbf{8 4 . 6}$ & 75.3 & 89.3 & 98.0 \\
VGG16 $^{[24]}$ & 75.3 & 74.0 & 79.0 & $\mathbf{8 4 . 2}$ & 90.7 & 99.3 \\
VisNet $^{[13]}$ & 73.7 & 78.0 & 79.0 & 82.0 & 90.7 & 99.0 \\
FCNN $_{\text {MOCNN }}^{[18]}$ & 84.4 & 77.3 & 82.2 & 82.4 & 90.4 & 98.7 \\
PORCNN & 84.7 & 80.9 & 79.1 & 82.9 & 93.1 & 98.2 \\
本文 & 80.0 & 81.5 & 84.2 & 80.3 & 91.2 & 98.5 \\
\hline
\end{tabular}

注. 加粗数值表示最好结果.

\section{5 结 语}

本文提出了一种端到端的深度学习算法, 进 行雾图像的能见度检测. 对于浓雾图像, 由于图像 结构信息不足, 挖掘和学习数据中隐藏的序数信 息非常重要. 该算法借助三元组相对学习来约束 网络的特征提取过程，利用序数关系使网络提取 的能见度属性在空间分布上满足序数规律, 最后 根据图像属性之间的相对位置关系，得到对待测 样本的能见度估计. 实验表明, 基于序数关系和相 对学习的能见度检测算法比现有的基于分类的深 度学习能见度算法更有效, 对训练样本数据量的 依赖更少. 同时，本文算法结果能够满足公路能见 度预警、交通控制等领域的实际应用需求.

\section{参考文献(References):}

[1] Liu Haibo, Yang Jie, Wu Zhengping, et al. A fast single image dehazing method based on dark channel prior and retinex the-
ory[J]. Acta Automatica Sinica, 2015, 41(7): 1264-1273(in Chinese)

(刘海波, 杨杰, 吴正平, 等. 基于暗通道先验和 Retinex 理 论的快速单幅图像去雾方法 [J]. 自动化学报, 2015, 41(7): 1264-1273)

[2] Chen Zhaozheng, Zhou Qingkui, Chen Qimei. Video visibility detection algorithm based on wavelet transformation[J]. Chinese Journal of Scientific Instrument, 2010, 31(1): 92-98(in Chinese)

(陈钊正，周庆逆，陈启美. 基于小波变换的视频能见度检 测算法研究与实现[J]. 仪器仪表学报, 2010, 31(1): 92-98)

[3] Kim K W. Estimation of visibility using a visual image[J]. Environmental Monitoring and Assessment, 2015, 187(3): Article No.66

[4] Babari R, Hautière N, Dumont É, et al. Visibility monitoring using conventional roadside cameras-Emerging applications [J]. Transportation Research Part C: Emerging Technologies, 2012, 22: $17-28$

[5] Carretas F, Wagner F, Janeiro F M. Atmospheric visibility and Angström exponent measurements through digital photography[J]. Measurement, 2015, 64:147-156

[6] Graves N, Newsam S. Using visibility cameras to estimate atmospheric light extinction[C] //Proceedings of the IEEE Workshop on Applications of Computer Vision. Los Alamitos: IEEE Computer Society Press, 2011: 577-584

[7] He K M, Sun J, Tang X O. Single image haze removal using dark channel prior[J]. IEEE Transactions on Pattern Analysis and Machine Intelligence, 2011, 33(12): 2341-2353

[8] Tarel J P, Hautière N, Caraffa L, et al. Vision enhancement in homogeneous and heterogeneous fog[J]. IEEE Intelligent Transportation Systems Magazine, 2012, 4(2): 6-20

[9] Gu J X, Wang Z H, Kuen J, et al. Recent advances in convolutional neural networks[J]. Pattern Recognition, 2018, 77: 354-377

[10] Li S Y, Fu H, Lo W L. Meteorological visibility evaluation on webcam weather image using deep learning features[J]. International Journal of Computer Theory and Engineering, 2017, 2017, 9(6): 455-461

[11] Krizhevsky A, Sutskever I, Hinton G E. ImageNet classification with deep convolutional neural networks[J]. Communications of the ACM, 2017, 60(6): 84-90

[12] Giyenko A, Palvanov A, Cho Y I. Application of convolutional neural networks for visibility estimation of CCTV images[C] //Proceedings of the International Conference on Information Networking. Los Alamitos: IEEE Computer Society Press, 2018: 875-879

[13] Palvanov A, Cho Y I. VisNet: deep convolutional neural networks for forecasting atmospheric visibility[J]. Sensors, 2019, 19(6): 1343

[14] Parikh D, Grauman K. Relative attributes[C] //Proceedings of the International Conference on Computer Vision. Los Alamitos: IEEE Computer Society Press, 2011: 503-510

[15] Sun B Y, Li J Y, Wu D D, et al. Kernel discriminant learning for ordinal regression[J]. IEEE Transactions on Knowledge and Data Engineering, 2010, 22(6) : 906-910

[16] Fouad S, Tino P. Adaptive metric learning vector quantization for ordinal classification[J]. Neural Computation, 2012, 24(11): 2825-2851

[17] Kramer S, Widmer G, Pfahringer B, et al. Prediction of ordinal 
classes using regression trees[J]. Fundamenta Informaticae, 2001, 47(1/2): 1-13

[18] Niu Z X, Zhou M, Wang L, et al. Ordinal regression with multiple output $\mathrm{CNN}$ for age estimation[C] //Proceedings of the IEEE Conference on Computer Vision and Pattern Recognition. Los Alamitos: IEEE Computer Society Press. 2016: 4920-4928

[19] Fu H, Gong M M, Wang C H, et al. Deep ordinal regression network for monocular depth estimation[C] //Proceedings of the IEEE/CVF Conference on Computer Vision and Pattern Recognition. Los Alamitos: IEEE Computer Society Press, 2018: 2002-2011

[20] Coh C K, Liu Y Z, Kong A W K. A constrained deep neural network for ordinal regression[C] //Proceedings of the IEEE Conference on Computer Vision and Pattern Recognition. Los Alamitos: IEEE Computer Society Press, 2018: 831-839

[21] Souri Y, Noury E, Adeli E. Deep relative attributes[C] //Proceedings of Asian Conference on Computer Vision. Heidelberg: Springer, 2016: 118-133

[22] Li S X, Shan S G, Chen X L. Relative forest for attribute prediction[C] //Proceedings of Asian Conference on Computer Vision. Heidelberg: Springer, 2012: 316-327

[23] Schroff F, Kalenichenko D, Philbin J. FaceNet: a unified embedding for face recognition and clustering $[\mathrm{C}] / /$ Proceedings of the IEEE Conference on Computer Vision and Pattern Recog- nition. Los Alamitos: IEEE Computer Society Press, 2015: 815-823

[24] Simonyan K, Zisserman A. Very deep convolutional networks for large-scale image recognition[OL]. [2020-02-26]. https:// arxiv.org/abs/1409.1556

[25] Ioffe S, Szegedy C. Batch normalization: accelerating deep network training by reducing internal covariate shift[OL]. [2020-02-26]. https:// arxiv.org/abs/1502.03167

[26] Srivastava N, Hinton G, Krizhevsky A, et al. Dropout: a simple way to prevent neural networks from overfitting[J]. Journal of Machine Learning Research, 2014, 15(1): 1929-1958

[27] Wen Y D, Zhang K P, Li Z F, et al. A discriminative feature learning approach for deep face recognition[C] //Proceedings of European Conference on Computer Vision. Heidelberg: Springer, 2016: 499-515

[28] Belaroussi R, Gruyer D. Impact of reduced visibility from fog on traffic sign detection[C] //Proceedings of the IEEE Intelligent Vehicles Symposium. Los Alamitos: IEEE Computer Society Press, 2014: 1302-1306

[29] Li Y C, Huang J F, Luo J B. Using user generated online photos to estimate and monitor air pollution in major cities[C] //Proceedings of the 7th International Conference on Internet Multimedia Computing and Service. New York: Association for Computing Machinery Press, 2015: Article No.79 